\title{
Dichotomous miR expression and immune responses following primary blood-stage malaria
}

\author{
Julie G. Burel, ${ }^{1,2}$ Simon H. Apte, ${ }^{1}$ Penny L. Groves, ${ }^{1}$ Michelle J. Boyle, ${ }^{3,4}$ Christine Langer, ${ }^{4}$ \\ James C. Beeson, ${ }^{4,5}$ James S. McCarthy, ${ }^{1,2}$ and Denise L. Doolan ${ }^{1,6}$ \\ ${ }^{1}$ QIMR Berghofer Medical Research Institute, Brisbane, Queensland, Australia. ${ }^{2}$ The University of Queensland, Brisbane, \\ Queensland, Australia. ${ }^{3}$ Menzies School of Health Research, Darwin, Northern Territory, Australia. ${ }^{4}$ Burnet Institute for \\ Medical Research and Public Health, Melbourne, Victoria, Australia. ${ }^{5}$ Department of Microbiology, Monash University, \\ Victoria, Australia. ${ }^{6}$ Centre for Biosecurity and Tropical Infectious Diseases, Australian Institute of Tropical Health and \\ Medicine, James Cook University, Cairns, Queensland, Australia.
}

\begin{abstract}
Clinical responses to infection or vaccination and the development of effective immunity are characterized in humans by a marked interindividual variability. To gain an insight into the factors affecting this variability, we used a controlled human infection system to study early immune events following primary infection of healthy human volunteers with blood-stage Plasmodium falciparum malaria. By day 4 of infection, a dichotomous pattern of high or low expression of a defined set of microRNAs (miRs) emerged in volunteers that correlated with variation in parasite growth rate. Moreover, high-miR responders had higher numbers of activated CD4+ $\mathrm{T}$ cells, and developed significantly enhanced antimalarial antibody responses. Notably, a set of 17 miRs was identified in the whole blood of low-miR responders prior to infection that differentiated them from high-miR responders. These data implicate preexisting host factors as major determinants in the ability to effectively respond to primary malaria infection.
\end{abstract}

Authorship note: J.G. Burel and S.H. Apte are co-first authors.

Conflict of interest: The authors have declared that no conflict of interest exists.

Submitted: February 14, 2017

Accepted: June 29, 2017

Published: August 3, 2017

\section{Reference information:}

JCI Insight. 2017;2(15):e93434.

https://doi.org/10.1172/jici.

insight.93434.

\section{Introduction}

The severity of clinical disease observed in humans during infection with many microorganisms appears to follow a Gaussian distribution. At each extremity is a minority that is either asymptomatic, or at risk of death, while the majority survive the infection with a spectrum of clinical severity. This type of interindividual variability is evident in responses to infection with most microbes that have plagued mankind including Plasmodium, influenza, and Mycobacterium tuberculosis. Malaria remains a global public health threat, with an estimated $40 \%$ of the population at risk and over 200 million clinical cases annually and it has an associated pattern of disease severity that ranges from asymptomatic to death (1). Although almost one-third of the world's population is infected with Mycobacterium tuberculosis, only around 5\% will progress to active disease in the absence of predisposing conditions, with an ultimate mortality rate of around $2 \%$ when untreated $(2,3)$. In the case of viral infections, the 1918 Spanish influenza pandemic that resulted in approximately 50 million deaths had a mortality rate of less than $10 \%$ in the most susceptible age groups, while the majority of those apparently infected displayed a spectrum of disease severity (4). This pattern of interindividual variability has been recognized since the early 20th century when Charles Nicolle first established the idea of inapparent infections $(5,6)$.

Understanding the mechanisms underlying this pattern of interindividual variability would facilitate the development of therapeutic interventions that could shift the curve, reducing disease burden and saving lives. Ultimately, the variability is shaped by the effectiveness of the immune response (which is the complex combination of many factors including environmental exposure and genetics) (7) and variability in the infecting microorganism.

In the case of malaria, interpreting or understanding the diversity of immune responses under conditions of natural exposure is difficult because initial exposure and subsequent reexposure initiates and amplifies the development of host immunity, which contributes to a lessened disease severity. This is further complicated by other confounders, such as parasite coinfections and genetic variability of the host and 
the parasite. A body of historical data from neurosyphilis patients treated with malaria therapy suggests that the interindividual variation in clinical responses observed in the field is also evident in more controlled conditions $(8,9)$. Clinical observations in those studies, where the infection was allowed to follow its natural course, tended to correspond to observations in the field, where some people had relatively few symptoms, most people were ill but controlled the infection, and some were very seriously ill and required drug treatment (8). Qualitative and quantitative interindividual variability in innate immune responses, which correlated with parasitological responses and clinical outcomes, have also been reported in individuals experimentally infected with Plasmodium falciparum sporozoites (10).

In the study reported herein, we took advantage of a highly controlled human blood-stage infection (CHMI) model utilizing the intravenous injection of $P$. falciparum-parasitized erythrocytes into malarianaive volunteers followed by antimalarial chemotherapy (11). This model affords a unique opportunity to study the first molecular events occurring following primary exposure of the human immune system to the Plasmodium parasite (12). Moreover, since the infecting dose and the nature of parasite inoculum are highly controlled and consistent, the immune responses to infection can be compared among individuals to define interindividual variability in immunity and susceptibility to infection. Using this model, we examined early immune responses in malaria-naive human volunteers upon primary infection with $P$. falciparum bloodstage parasites, with emphasis on the detection and nature of response diversity. A principal objective was to examine microRNA (miR) expression profiles in volunteers before infection and at day 4 and day 7 of infection. miRs are emerging as important regulators of the immune response: they are abundant short noncoding RNAs that provide posttranscriptional modulation of gene expression by binding mRNA and blocking ribosomal translation and increasing mRNA degradation rate $(13,14)$. It is estimated they target approximately $60 \%$ of all human genes with high promiscuity, where each miR may have many thousands of different mRNA targets. They are recognized as important regulators of biological processes including $\mathrm{T}$ cell- and B cell-mediated immune responses and other immune functions $(13,14)$. Studies of natural infection with influenza, Mycobacterium tuberculosis, and HIV have suggested that miR profiling studies can identify biomarkers of infection and predictors of clinical outcome (15-18). However, the expression profile of host miRs during Plasmodium infection in humans has not yet been investigated and their ability to act as biomarkers for infection and/or immune control remains unknown.

Herein, we report the identification of an unexpected dichotomy in the whole-blood miR expression profile of CHMI volunteers as early as day 4 of infection: approximately $50 \%$ of individuals upregulated a set of miRs involved in immune responses (high-miR responders), whereas the remaining volunteers downregulated these miRs (low-miR responders). Covariate analysis of parasite growth rate and $\mathrm{T}$ cell activation over the first 7 days, or antibody responses at day 28 after infection, showed that the dichotomous nature of the miR response is functionally reflected in the ability to control the parasite and to mount a protective immune response. Finally, categorizing volunteers into groups of high- or low-miR responders, we identified a distinct pattern of miR and mRNA expression evident in the blood of low-responder volunteers prior to infection, suggesting that the response to infection was predetermined and may be predictable. Our results suggest that the ability to mount effective anti-Plasmodium immune responses may be relatively impaired in approximately $50 \%$ of the naive population and that miR expression profiles are useful biomarkers to understand and predict this impairment. This is, to our knowledge, the first report in any system of a distinct dichotomy in cellular immune responses in humans and has important practical implications in understanding the nature of interindividual variability in clinical responses to infection and vaccination and induction of effective antipathogen immunity.

\section{Results}

Dichotomy in miR expression profile generated in humans following primary exposure to $P$. falciparum. To define specific host miRs expressed during the primary immune response to $P$. falciparum blood-stage infection in humans, we employed a targeted quantitative reverse transcription PCR (RT-qPCR) strategy on total RNA extracted from whole blood using the miScript miRNA PCR Array system to measure the expression of 84 miRs known to be associated with $\mathrm{T}$ cell and B cell activation. Whole-blood samples were collected from 14 randomly selected malaria-naive volunteers in 3 independent cohorts prior to and on day 4 and day 7 of infection with $P$. falciparum-infected erythrocytes (demographics available in Supplemental Table 1; supplemental material available online with this article; https://doi.org/10.1172/jci. insight.93434DS1). Out of the $84 \mathrm{miRs}$ assessed, a set comprising $71 \mathrm{miRs}$ was reproducibly detectable 
A
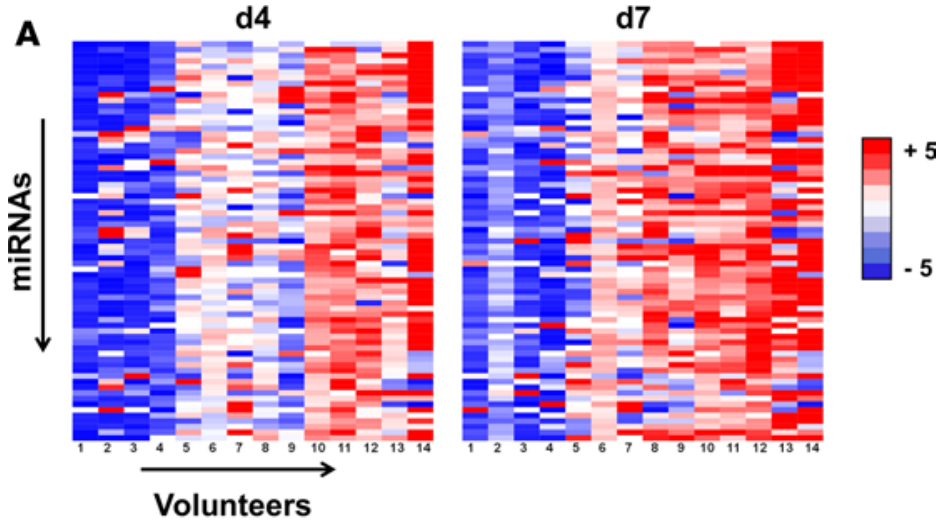

Figure 1. Whole-blood miR expression profile in malaria-naive human volunteers experimentally infected with blood-stage $P$. falciparum. Whole blood was collected prior to, and on days 4 and 7 of infection in PAXgene blood RNA tubes. Relative quantification of 71 miRs involved in T cell and B cell activation detected at day 4 (d4) and day 7 (d7) of infection (compared to day 0 ) was determined by RTqPCR using human $T$ cell and $B$ cell activation miScript miRNA PCR arrays (Qiagen) and the ddCt method. (A) Heatmaps representing the

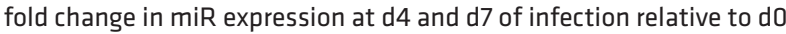
for each miR across 14 volunteers from 3 independent cohorts. miRs were sorted according to their order within the miScript PCR array layout. (B) Correlation for each volunteer between the sum of fold changes for each of the 71 miRs' expression at $\mathrm{d} 4$ or $\mathrm{d} 7$ after infection (relative to d0), determined using Pearson's correlation test.

and each of the individual $71 \mathrm{miRs}$ showed marked variation in fold-change expression in individual volunteers prior to and following infection (Figure 1A and Supplemental Table 2 ). When compared with their expression levels prior to infection, approximately $50 \%$ of volunteers upregulated almost all of the $71 \mathrm{miRs}$ included in the set following infection (highmiR responders), while the remaining volunteers downregulated most of the miRs contained within the set (low-miR responders). Expression of each of the miRs in each volunteer was highly correlated between day 4 and day 7 of infec-

tion (Figure 1B and Supplemental Table 2), suggesting that the miR expression pattern generated upon infection within each volunteer was stable in the peripheral circulation over time.

Early increases in expression of a subset of miRs following blood-stage infection associates with reduced parasite burden. To determine if an association exists between miR expression and $P$. falciparum replication, we investigated the correlation between the miR expression profile following infection and the blood-stage parasite burden observed for each volunteer. Total parasite burden during the first 7 days of infection was calculated as the area under the curve (AUC) based on the parasitemia as measured by qPCR from day 0 to day 7 of infection.

Of the 71 miRs detected in the peripheral blood of volunteers, there was a significant correlation between blood-stage parasite burden and the fold-change expression at either day 4 or day 7 of infection, or both, for 16 miRs (Table 1). Importantly, all were negative correlations, suggesting that increased levels of these miRs are associated with a reduction in parasite growth rate. In particular, the levels of 3 miRs (miR30e-5p, miR30c-5p, and miR15a-3p) observed at both day 4 and day 7 of infection strongly inversely correlated with parasite burden (correlation coefficients $r=-0.855$ and $r=-0.691$ for miR30e-5p, $r=$ -0.867 and $r=-0.682$ for miR30c-5p, $r=-0.816$ and $r=-0.632$ for miR15a-3p, at day 4 and day 7 , respectively; Table 1). When looking at Bonferroni-corrected $P$ values, these 3 miRs were also the only miRs whose expression (at day 4 but not day 7 of infection) was significantly inversely correlated with parasite burden (Table 1).

Correlation between the expression of miR15a-3p, miR30c-5p, and miR30e-5p during infection and blood-stage parasite burden was validated using specific RT-qPCR assays for miR (Taqman). Expression of miR15a-3p, miR30c-5p, and miR30e-5p was measured in total RNA isolated from whole blood collected prior to infection, and at day 4 and day 7 of infection, in the same 14 individuals assayed using the miScript miRNA PCR Arrays (as above) as well as an additional 7 individuals (two from Cohort 1, one from Cohort 2, and four from an additional fourth Cohort), for a total of 21 volunteers from 4 independent cohorts (demographics available in Supplemental Table 1). These results validated the initial observations: for each of the 21 volunteers, the average expression of the 3 individual miRs at day 4 and day 7 of infection was significantly inversely correlated with the overall parasite burden from day 0 to day 7 of infection (Figure $2 \mathrm{~A}$ ), as too was the average of fold changes for the 3-miR signature (Figure $2 \mathrm{~B}$ ).

A 3-miR signature develops early during infection that distinguishes high-and low-miR responders. The 3-miR signature (miR15a-3p, miR30c-5p, and miR30e-5p) could successfully distinguish high-miR responders and low-miR responders: high-miR responders had an average fold change in expression of $\mathrm{miR} 15 \mathrm{a}-3 \mathrm{p}$, 
Table 1. Correlation between parasite burden and the relative miR expression at day 4 and day 7 of infection with blood-stage P. falciparum in malaria-naive volunteers

\begin{tabular}{|c|c|c|c|c|c|c|}
\hline & \multicolumn{3}{|c|}{ Day 4} & \multicolumn{3}{|c|}{ Day 7} \\
\hline & \multirow{2}{*}{ Pearson $r$} & \multicolumn{2}{|c|}{$P$ value } & \multirow{2}{*}{ Pearson $r$} & \multicolumn{2}{|c|}{$P$ value } \\
\hline & & Raw & Bon $^{A}$ & & Raw & Bon $^{A}$ \\
\hline hsa-miR-30e-5p & -0.86 & $<0.0001$ & 0.007 & -0.65 & 0.013 & 0.79 \\
\hline hsa-miR-15a-3p & -0.82 & 0.0004 & 0.03 & -0.69 & 0.006 & 0.37 \\
\hline hsa-miR-92a-3p & -0.81 & 0.001 & 0.07 & -0.30 & 0.30 & 1 \\
\hline hsa-miR-26a-5p & -0.67 & 0.009 & 0.64 & -0.12 & 0.69 & 1 \\
\hline hsa-miR-27a-3p & -0.66 & 0.019 & 1 & -0.76 & 0.007 & 0.43 \\
\hline hsa-miR-15a-5p & -0.62 & 0.024 & 1 & -0.68 & 0.011 & 0.67 \\
\hline hsa-miR-342-3p & -0.61 & 0.022 & 1 & -0.06 & 0.83 & 1 \\
\hline hsa-miR-181b-5p & -0.60 & 0.03 & 1 & -0.11 & 0.72 & 1 \\
\hline hsa-miR-331-3p & -0.39 & 0.17 & 1 & -0.54 & 0.04 & 1 \\
\hline
\end{tabular}

miR30c-5p, and miR30e-5p greater than 1.5 ( $n=11$ volunteers; average fold-change expression of 5.9, 2.6, and 3.7 for miR15a-3p, miR30c-5p, and miR30e-5p, respectively); and low miR responders had an overall fold change in miR expression less than or equal to 1 ( $n=9$ volunteers; average fold-change expression of $0.79,0.78$, and 0.81 for miR15a-3p, miR30c-5p, and miR30e-5p, respectively) (Figure $2 \mathrm{C}$ ). One volunteer whose overall fold change was between 1 and 1.5 was not classified as a high- or low-miR responder, and was therefore excluded from subsequent analysis. Thus, in this model, the levels of miR15a-3p, miR30c-5p, and $\mathrm{miR} 30 \mathrm{e}-5 \mathrm{p}$ in the peripheral blood constitute a 3-miR biomarker that associates with ability to control blood-stage parasite growth.

High-miR responders have increased numbers of activated $C D 4^{+} T$ cells during infection. Next, we investigated whether high- and low-miR responders could be distinguished on the basis of the quality and frequency of $\mathrm{T}$ cells and $\mathrm{B}$ cells circulating in the peripheral blood during infection. Firstly, no differences were evident in the total white blood cell count when comparing the high- and low-miR responder groups either at day 0 or day 7 of infection; however, high-miR responders (but not low-miR responders) displayed a significant reduction in the total lymphocyte count from day 0 to day 7 of infection (Figure 3A). Cell subsets were further assessed by flow cytometry. Changes in the total $\mathrm{CD} 4^{+} \mathrm{T}$ cell, $\mathrm{CD} 8^{+} \mathrm{T}$ cell, and $\mathrm{CD} 19^{+} \mathrm{B}$ cell counts from day 0 to day 7 of infection positively correlated with parasite burden and inversely correlated with miR expression (Figure 3B), although only the correlation between miR expression and changes in $\mathrm{CD}^{+}$ T cell counts was statistically significant (Spearman's test). This is consistent with sequestration of these cells from the peripheral circulation into tissues. T and B cells expressing the early activation marker CD69 increased numerically from day 0 to day 7 of infection for both the low- and high-miR responder groups, but with a higher magnitude in the latter group (Figure 3C). More notably, high-miR responders displayed an increase in the number of $\mathrm{CD} 4^{+} \mathrm{T}$ cells coexpressing the activation/proliferation markers $\mathrm{CD} 38$ and Ki67 that was not evident for $\mathrm{CD}^{+} \mathrm{T}$ cells or B cells or for the low-miR responders (Figure 3D). Thus, although high-miR responders have an apparent reduction in peripheral lymphocyte numbers, they have a specific increase in the number of circulating activated $\mathrm{CD} 4^{+} \mathrm{T}$ cells. These findings are consistent with our recent study reporting significant association between the expansion of $\mathrm{CD} 38^{+} \mathrm{CD} 4^{+} \mathrm{T}$ cells and reduced parasite burden in the P. falciparum CHMI model (19).

High-miR responders mount a more robust anti-P. falciparum antibody response characterized by increased cytophilic subclass induction. Having determined that high-miR responders appear to mount a stronger $\mathrm{CD}^{+} \mathrm{T}$ cell-mediated immune response during $P$. falciparum infection, we next assessed whether high- 
A

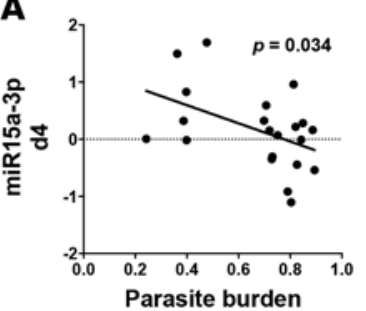

Parasite burden

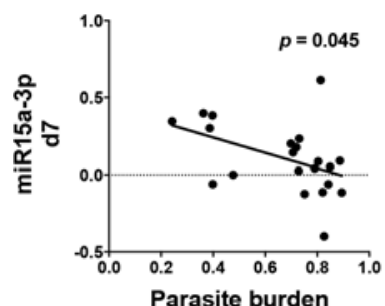

Parasite burden

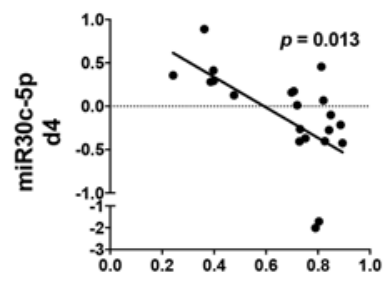

Parasite burden

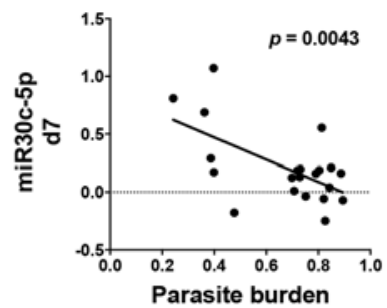

Low miRNA responders
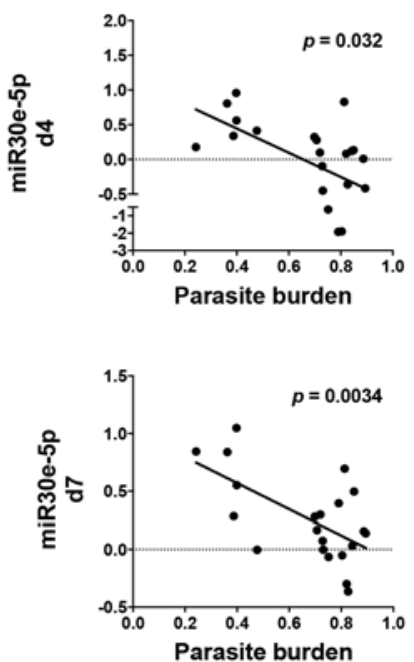

High miRNA responders
B
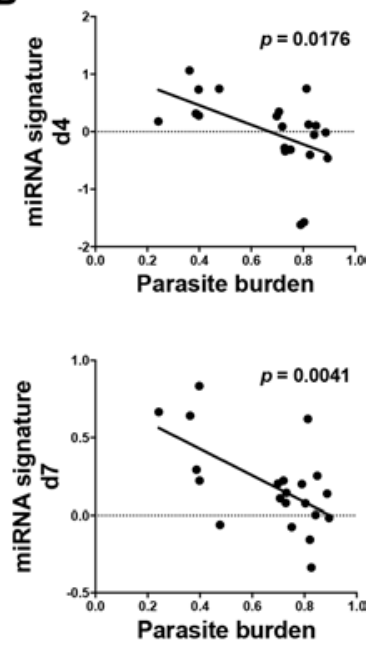

$+5$

Fold

change

Average (d4-d7)
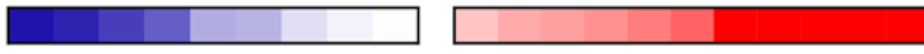

\section{$-5$}

Figure 2. Expression of miR15a-3p, miR30c-5p, and miR30e-5p during $P$. falciparum blood-stage infection inversely correlates with parasite burden. Whole blood was collected prior to, and on days 4 and 7 of infection into PAXgene blood RNA tubes. Relative quantification of miR15a-3p, miR30c-5p, and miR30e-5p at day 4 (d4) and day 7 (d7) of infection (compared with day 0 ) was determined by RT-qPCR using Taqman microRNA assays and the ddCt method. Total parasite burden during the first 7 days of infection was defined as the AUC of the log-transformed parasite levels measured using a consensus $P$. falciparum qPCR assay from day 0 to day 7 of infection. Correlation between parasite burden and the relative average expression of miR15a-3p, miR30c-5p, and miR30e5p (A) at day 4 or day 7 of infection or (B) the average of fold changes for miR15a-3p, miR30c-5p, and miR30e-5p (miR signature). Parasite burden and miR expression datasets were log transformed for graphic representation and linear regression analysis. (C) Volunteers were classified as low-miR responders ( $n=9$ volunteers, average fold change $\leq 1)$ or high-miR responders ( $n=11$ volunteers, average fold change $\geq 1.5$ ) according to the combined relative expression of miR15a-3p, miR30c-5p, and miR30e-5p at day 4 and day 7 of infection. One volunteer whose overall fold change was between 1 and 1.5 was not classified as a high- or low-miR responder, and was therefore excluded. Graphs show data from 21 volunteers (each depicted as a colored square across the $x$ axis) from 4 independent cohorts.

and low-miR responders differ with regard to the acquisition of antibody responses after infection. Antibodies are thought to mediate protection against blood-stage malaria by inhibiting merozoite invasion of erythrocytes and by enhancing opsonic phagocytosis of exoerythrocytic merozoites (reviewed in ref. 20). In particular, cytophilic subclass antibodies (IgG1 and IgG3) against merozoite surface protein 1 (MSP1) and MSP2, the 2 most abundant merozoite antigens (21), have been associated with protection from malaria in longitudinal cohort studies in children in malaria endemic regions (22-24). Accordingly, we assessed parasite-specific and antigen-specific antibody responses in our cohort of volunteers to ascertain if differences existed between the responses elicited in high-miR responders when compared with low responders.

A significant increase in IgG antibody titer against total blood-stage parasite extract was detected 28 days following infection in the plasma of high-miR responders but not low-miR responders (Figure 4A). Enhanced IgG antibody responses specific to Plasmodium merozoite proteins MSP1-42 (42-kDa fragment of MSP1) and MSP2 were also observed in the high-miR responders (Figure 4B). For MSP2 antibody responses, there was sufficient induction of IgG antibodies to analyze the subclass composition of the response. For the low-miR responders, there was induction of IgG1, IgG2, and IgG4 antibodies, but not IgG3 responses. For high-miR responders, all subclasses, including IgG3 antibodies were induced (Figure 4C), and the fold change of induction was significantly greater for IgG1 and IgG3 in the high-miR responders (Figure 4D). We also assessed the breadth of the antibody response by measuring the induction of $\operatorname{IgM}$ antibodies against a panel of merozoite surface antigens. When compared with 
Table 2. Correlation between cellular and humoral immune responses in high- and low-miR responders

\begin{tabular}{|c|c|c|c|c|c|c|c|c|c|}
\hline \multirow[b]{3}{*}{ Protein } & \multirow[b]{3}{*}{ Ig subclass } & \multicolumn{8}{|c|}{ High-miR responders } \\
\hline & & \multicolumn{2}{|c|}{$\mathrm{CD}^{+}{ }^{+} \mathrm{T}$ cells $\mathrm{CD} 8^{+} \mathrm{Ki}^{-} 7^{+} \mathrm{dO}-\mathrm{d} 7$} & \multicolumn{2}{|c|}{$\mathrm{CD4}^{+} \mathrm{T}$ cells $\mathrm{CD69}^{+} \mathrm{d0}-\mathrm{d} 7$} & \multicolumn{2}{|c|}{$\mathrm{CD8}^{+} \mathrm{T}$ cells $\mathrm{CD69}^{+} \mathrm{d0}-\mathrm{d} 7$} & \multicolumn{2}{|c|}{ CD19 + B cells CD69+ d0 $-d 7$} \\
\hline & & Spearman $r$ & $P$ value & Spearman $r$ & $P$ value & Spearman $r$ & $P$ value & Spearman $r$ & $P$ value \\
\hline MSP1 & IgM & 0.01 & $>0.9999$ & 0.83 & 0.005 & 0.47 & 0.18 & 0.73 & 0.02 \\
\hline MSP1-42 & $\lg C$ & 0.31 & 0.39 & 0.50 & 0.14 & 0.22 & 0.54 & 0.42 & 0.23 \\
\hline \multirow[t]{4}{*}{ MSP2 } & IgG & -0.10 & 0.79 & 0.71 & 0.03 & 0.44 & 0.20 & 0.50 & 0.14 \\
\hline & $\lg \mathrm{G} 1$ & -0.50 & 0.14 & 0.73 & 0.02 & 0.56 & 0.10 & 0.56 & 0.10 \\
\hline & $\operatorname{lgG4}$ & -0.27 & 0.45 & 0.67 & 0.04 & 0.47 & 0.18 & 0.76 & 0.01 \\
\hline & $\lg M$ & -0.10 & 0.79 & 0.85 & 0.003 & 0.52 & 0.13 & 0.75 & 0.02 \\
\hline MSP3 & $\operatorname{Ig} M$ & 0.37 & 0.30 & 0.45 & 0.19 & 0.16 & 0.66 & 0.22 & 0.54 \\
\hline MSP4 & IgM & 0.20 & 0.58 & 0.49 & 0.15 & 0.48 & 0.17 & 0.28 & 0.43 \\
\hline MSP5 & IgM & 0.44 & 0.20 & 0.49 & 0.15 & 0.38 & 0.28 & 0.36 & 0.31 \\
\hline Protein & Ig subclass & Spearman $r$ & $P$ value & Spearman $r$ & $P$ value & Spearman $r$ & $P$ value & Spearman $r$ & $P$ value \\
\hline MSP1 & IgM & 0.65 & 0.07 & -0.23 & 0.55 & -0.28 & 0.46 & -0.37 & 0.34 \\
\hline MSP1-42 & IgG & 0.20 & 0.61 & -0.10 & 0.81 & -0.13 & 0.74 & -0.25 & 0.52 \\
\hline \multirow[t]{6}{*}{ MSP2 } & $\lg G$ & 0.50 & 0.18 & -0.13 & 0.74 & -0.22 & 0.58 & -0.32 & 0.41 \\
\hline & IgG1 & 0.10 & 0.81 & -0.25 & 0.52 & -0.27 & 0.49 & -0.38 & 0.31 \\
\hline & $\lg C 2$ & 0.37 & 0.34 & -0.10 & 0.81 & -0.15 & 0.71 & -0.22 & 0.58 \\
\hline & IgG3 & 0.22 & 0.58 & -0.48 & 0.19 & -0.53 & 0.15 & -0.62 & 0.09 \\
\hline & $\lg C 4$ & 0.28 & 0.46 & -0.10 & 0.81 & -0.20 & 0.61 & -0.30 & 0.44 \\
\hline & IgM & 0.32 & 0.41 & -0.05 & 0.91 & -0.17 & 0.68 & -0.28 & 0.46 \\
\hline MSP3 & IgM & 0.20 & 0.61 & 0.47 & 0.21 & 0.50 & 0.18 & 0.42 & 0.27 \\
\hline MSP4 & IgM & 0.35 & 0.36 & 0.17 & 0.68 & 0.08 & 0.84 & -0.05 & 0.91 \\
\hline
\end{tabular}

MSP, merozoite surface protein; PfSE, $P$. falciparum schizont extract.

low-miR responders, high-miR responders increased the induction of $\operatorname{IgM}$ antibodies against MSP1, MSP2, MSP4, and MSP5 (Figure 4E).

These observations show that high-miR responders mount a more robust and broader antibody response than low-miR responders, and this response has been associated in several studies with protection against malaria. It is unlikely that these antibody responses (observed at 28 days after infection, 21 days after drug cure) would be responsible for the early control of parasite burden up to day 7, but our data do suggest that high-miR responders may become immune to clinical disease more rapidly than low responders. In line with this hypothesis, we found significant positive correlations between the frequency of activated $\mathrm{CD} 69^{+} \mathrm{CD} 4^{+} \mathrm{T}$ cells at day 7 of infection and antigen-specific antibody responses at day 28 of infection for high-miR responders, but not for low-miR responders (Table 2).

$m R N A$ expression further defines high- and low-miR responders before and during infection. Above, we defined high- and low-miR responders based on early changes in miR expression observed prior to and on day 4 or day 7 of infection. One of the principal mechanisms by which miR exerts its activity is to bind and destabilize mRNA, inducing increased degradation (13). Hence, we reasoned that mRNA levels may be differentially affected between high- and low-miR responders. Accordingly, we utilized the NanoString technology platform to assess the mRNA expression levels of 135 genes known to be associated with $\mathrm{T}$ and B cell function in whole-blood samples taken from randomly selected 6 high-responder, and 6 lowresponder volunteers, prior to (day 0 ) and on day 7 of infection. 
A WBC counts

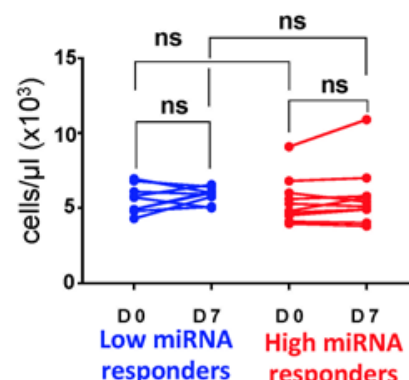

B

$\mathrm{CD4}^{+} \mathrm{T}$ cells

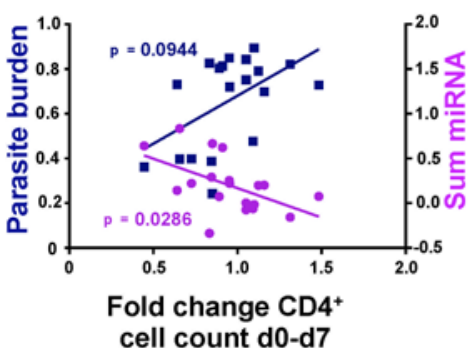

C

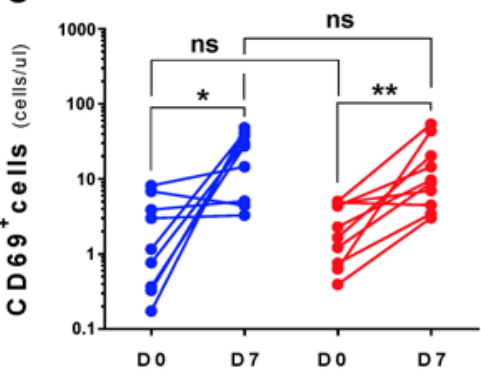

D

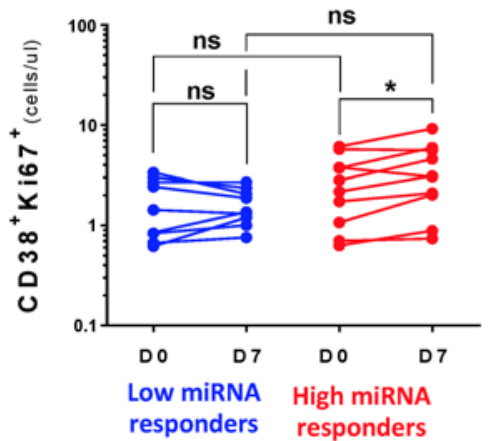

Lymphocyte counts

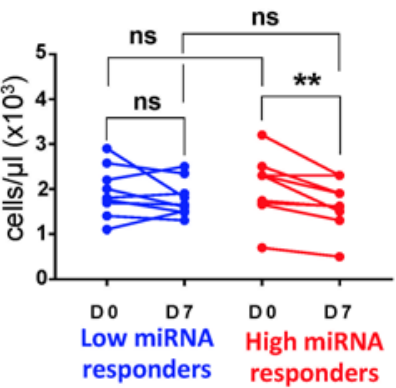

$\mathrm{CD}^{+} \mathrm{T}$ cells

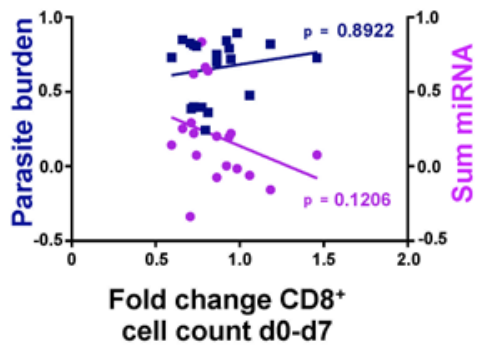

$\mathrm{CD8}^{+} \mathrm{T}$ cells
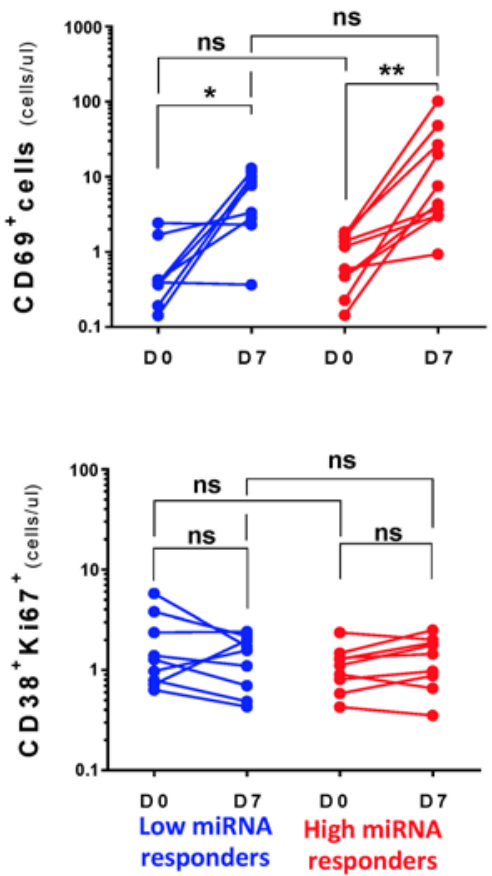

CD19+ $\mathrm{B}$ cells

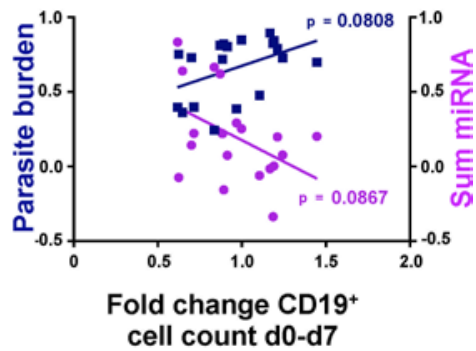

CD19+ $B$ cells
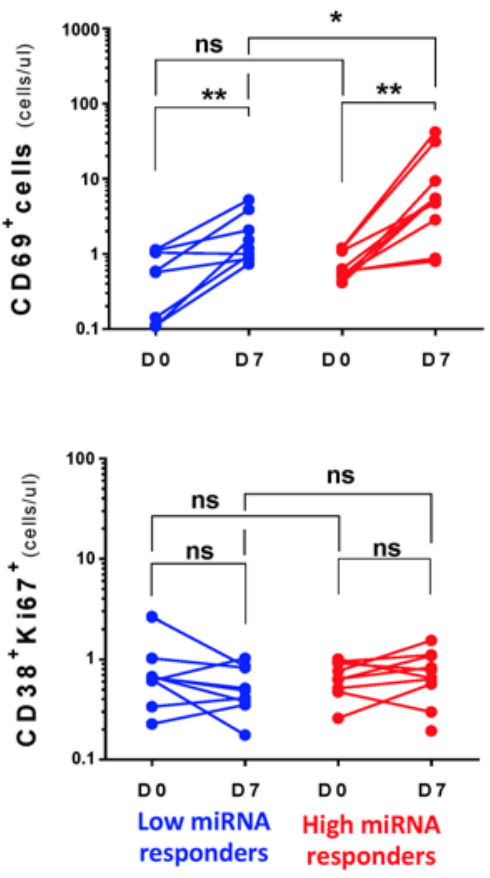

Figure 3. Increased $\mathrm{CD4}^{+} \mathbf{T}$ cell responses in high-miR responders between day $\mathbf{0}$ and day $\mathbf{7}$ of infection. Whole blood was collected prior to (d0), and on day 7 of infection (d7) from individuals classified as low-miR responders $(n=9)$ or high-miR responders $(n=11)$ as detailed in Figure 2C. (A) White blood cell (WBC) and lymphocyte counts determined on the day of collection by automated cell counter. (B) Correlation of CD4+, CD8 $8^{+}$, and $C D 19^{+}$cell counts in peripheral blood (determined by flow cytometry) and parasite burden over first 7 days of infection (left axis, square symbols), or the average (Avg) of fold changes (FC) for miR15a-3p, miR30c-5p, and miR30e-5p from do to d7 of infection (average miR, right axis, round symbols) (Spearman's correlation). The number of $\mathrm{CD}^{+}, \mathrm{CD}^{+}$, and $\mathrm{CD19}^{+}$cells expressing $\mathrm{CD69}$ (C), or coexpressing Ki67 and CD38 was determined directly ex vivo from whole-blood samples by flow cytometry. In $\mathbf{A}, \mathbf{C}$, and $\mathbf{D}$ the differences between $\mathrm{DO}$ and $\mathrm{D} 7 \mathrm{in}$ low-miR responders or high-miR responders were determined using the nonparametric paired Wilcoxon test; the comparison between the high- and low-responder groups on DO or on D7 was determined using nonparametric Mann-Whitney test. ${ }^{*} P<0.05$; ${ }^{* *} P<0.01$. Graphs show the data for 20 volunteers from 4 independent cohorts. ns, not significant. 
Table 3. Differentially expressed mRNAs between high- and low-miR responder groups

\begin{tabular}{|c|c|c|c|}
\hline \multicolumn{4}{|c|}{ Significant differences at day 0} \\
\hline \multirow[t]{2}{*}{ Gene } & \multicolumn{2}{|c|}{$P$ value } & \multirow[t]{2}{*}{ Log2 ratio } \\
\hline & Raw & Bon $^{A}$ & \\
\hline CD27 & 0.009 & 0.94 & 0.77 \\
\hline CCR7 & 0.009 & 0.94 & 0.74 \\
\hline CD28 & 0.009 & 0.94 & 0.60 \\
\hline CD226 & 0.009 & 0.94 & 0.55 \\
\hline ITK & 0.009 & 0.94 & 0.49 \\
\hline LCK & 0.009 & 0.94 & 0.38 \\
\hline CD40LG & 0.015 & 1 & 0.67 \\
\hline IL7R & 0.015 & 1 & 0.59 \\
\hline RUNX1 & 0.026 & 1 & 0.67 \\
\hline SOCS5 & 0.026 & 1 & 0.52 \\
\hline ICOS & 0.026 & 1 & 0.45 \\
\hline CD4 & 0.041 & 1 & 0.77 \\
\hline IRF4 & 0.041 & 1 & 0.57 \\
\hline IL2Ra & 0.041 & 1 & 0.57 \\
\hline ITCA4 & 0.041 & 1 & 0.47 \\
\hline CD96 & 0.041 & 1 & 0.46 \\
\hline S1PR1 & 0.041 & 1 & 0.45 \\
\hline \multicolumn{4}{|c|}{ Significant differences at day 7} \\
\hline \multirow[t]{2}{*}{ Gene } & \multicolumn{2}{|c|}{$P$ value } & Log2 ratio \\
\hline & Raw & Bon $^{A}$ & \\
\hline IL7R & 0.026 & 1 & 0.467 \\
\hline
\end{tabular}

${ }^{A}$ Bonferroni-corrected $P$ value. mRNA expression in whole blood was determined for a set of 135 genes using NanoString in 12 volunteers taken prior to (do), and on day 7s (d7) of infection the from high- and low-miR responder groups. All differentially expressed genes (Mann-Whitney test).
Table 4. Differentially expressed mRNAs between $\mathrm{dO}$ and $\mathrm{d} 7 \mathrm{of}$ infection

\begin{tabular}{|c|c|c|c|}
\hline \multicolumn{4}{|c|}{$\begin{array}{l}\text { Significant paired differences d0-d7 low-miR } \\
\text { responders }\end{array}$} \\
\hline \multirow[t]{2}{*}{ Gene } & \multicolumn{2}{|c|}{$P$ value } & \multirow[t]{2}{*}{ Log2 ratio } \\
\hline & Raw & $\mathrm{Bon}^{\mathrm{A}}$ & \\
\hline GFI1 & 0.031 & 1 & -0.51 \\
\hline TBX21 & 0.031 & 1 & -0.49 \\
\hline CD160 & 0.031 & 1 & -0.46 \\
\hline PRF1 & 0.031 & 1 & -0.42 \\
\hline GNLY & 0.031 & 1 & -0.40 \\
\hline GZMH & 0.031 & 1 & -0.40 \\
\hline KLRG1 & 0.031 & 1 & -0.26 \\
\hline MAF & 0.031 & 1 & -0.22 \\
\hline CCL5 & 0.031 & 1 & -0.20 \\
\hline CD96 & 0.031 & 1 & -0.18 \\
\hline IL27RA & 0.031 & 1 & -0.17 \\
\hline IL2RB & 0.031 & 1 & -0.17 \\
\hline NFATC2 & 0.031 & 1 & -0.15 \\
\hline CD226 & 0.03 & 1 & -0.14 \\
\hline IL32 & 0.031 & 1 & -0.12 \\
\hline \multicolumn{4}{|c|}{$\begin{array}{l}\text { Significant paired differences d0-d7 high-miR } \\
\text { responders }\end{array}$} \\
\hline \multirow[t]{2}{*}{ Gene } & \multicolumn{2}{|c|}{$P$ value } & \multirow[t]{2}{*}{ Log2 ratio } \\
\hline & Raw & Bon $^{A}$ & \\
\hline FAS & 0.031 & 1 & 0.82 \\
\hline JAK2 & 0.031 & 1 & 0.78 \\
\hline STAT5A & 0.031 & 1 & 0.59 \\
\hline JAK3 & 0.031 & 1 & 0.58 \\
\hline RUNX1 & 0.031 & 1 & 0.56 \\
\hline TCFB1 & 0.031 & 1 & 0.54 \\
\hline STAT5B & 0.031 & 1 & 0.53 \\
\hline MAF & 0.031 & 1 & 0.49 \\
\hline IL2RA & 0.031 & 1 & 0.39 \\
\hline IL21R & 0.031 & 1 & 0.34 \\
\hline CD40LG & 0.031 & 1 & 0.31 \\
\hline KLRB1 & 0.031 & 1 & -0.53 \\
\hline GZMA & 0.031 & 1 & -0.53 \\
\hline
\end{tabular}

${ }^{A}$ Bonferroni-corrected $P$ value. Paired analysis of changes in mRNA expression for individuals classified as low- or high-miR responders between $\mathrm{d} 0$ and $\mathrm{d} 7$ of infection (Wilcoxon test, $n=6$ subjects/group).

Prior to infection, 17 of the 135 genes were significantly differentially expressed between the high- and low-responder groups (Mann-Whitney test, raw $P$ value $<0.05)$, although none of the Bonferroni-corrected $P$ values reached significance. In all cases, mRNA expression was greater in the low-responder group prior to infection when compared with the high-responder group (Figure 5A, top 6 differentially expressed genes by raw $P$ value; and Table 3, all differentially expressed genes). By day 7 of infection, only 1 gene remained significantly differentially expressed (IL7R), although in many cases the trend remained.

Next, we performed a paired analysis for the set of 135 genes, comparing mRNA expression levels in each volunteer from day 0 to day 7 . In the low-responder group, 15 genes were significantly regulated between day 0 and day 7 and in all cases the mRNA was downregulated (Wilcoxon test, raw $P$ value $<0.05$ ) (Figure 5B and Table 4). This included only 2 genes that were differentially expressed between the groups prior to infection (CD226 and CD96) (compare Tables 3 and 4). In contrast, 13 genes were significantly regulated in the high-responder group, 11 of which were upregulated from day 0 to day 7 (Figure $5 \mathrm{C}$ and 
A

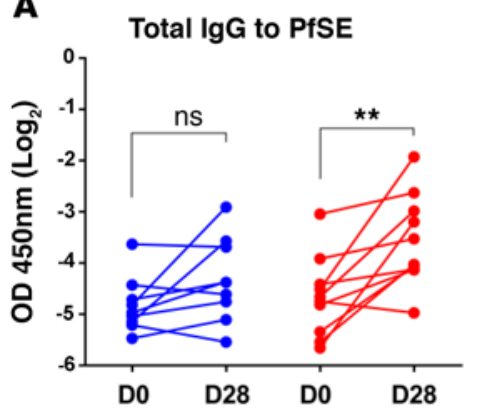

B

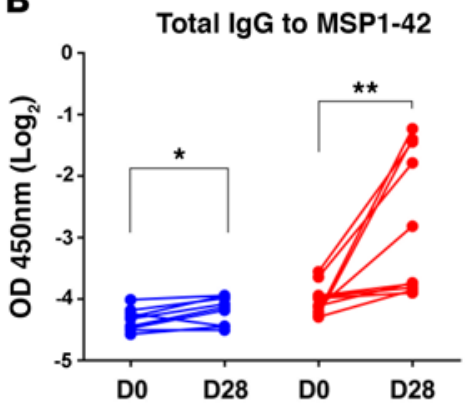

Total IgG to MSP2

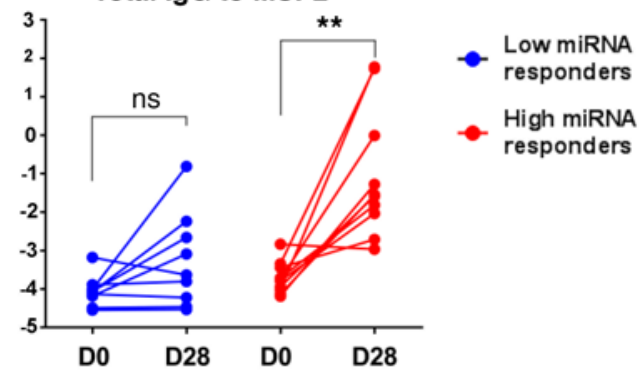

C

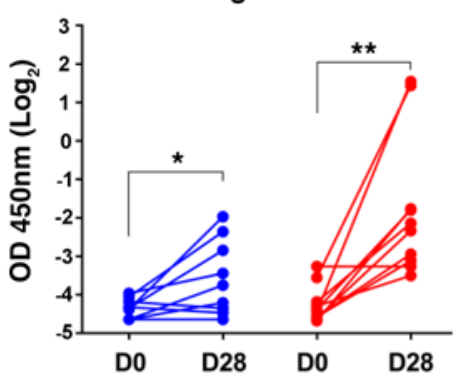

Total lgG2 to MSP2

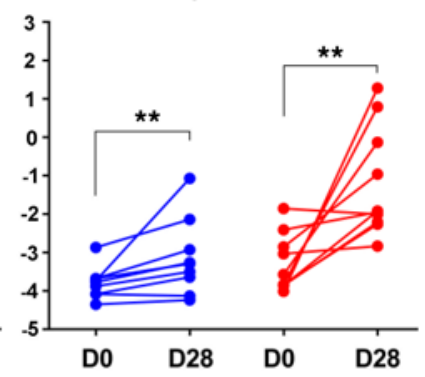

Total lgG3 to MSP2

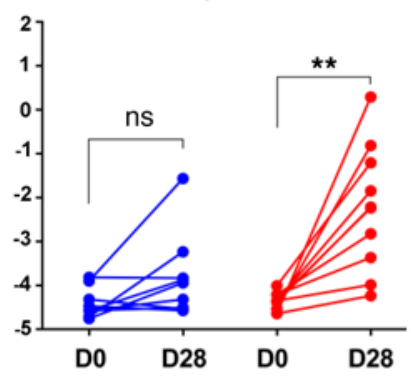

Total IgG4 to MSP2

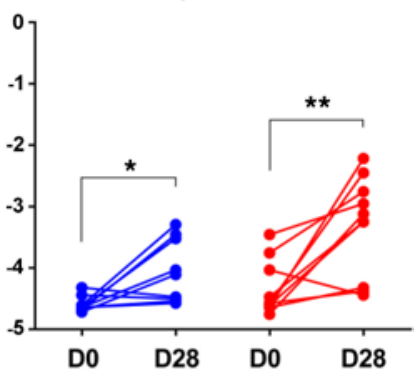

D

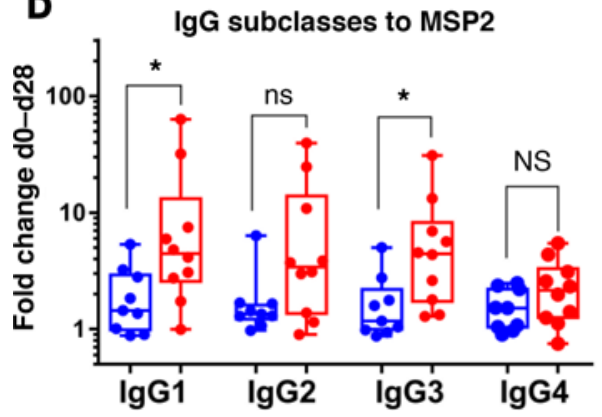

E

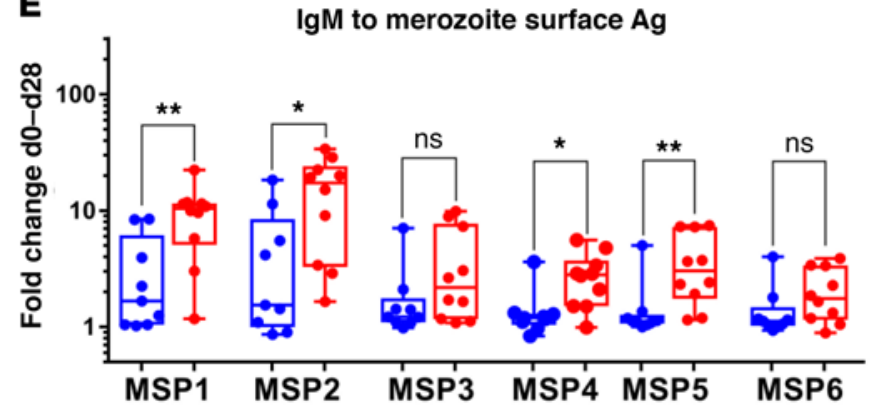

Figure 4. The anti-Plasmodium antibody response is more robust in high-miR responders. IgG antibody titers specific to (A) $P$. falciparum schizont extract (PfSE) or (B) MSP1-42 (42-kDa fragment of merozoite surface 1 [MSP1]) and MSP2 P. falciparum proteins were determined using an ELISA assay in plasma samples collected prior to infection and $28 \pm 3$ days after the day of infection. (C) IgC subclass responses (IgG1, IgG2, IgG3, and IgG4) were determined for MSP2. (D) Fold-change induction between d0 and d28 in antibody titers was calculated for each subclass against MSP2 and was compared between low- and high-miR responders. (E) IgM fold-change induction between d0 and d28 in response to MSP1, MSP2, MSP3, MSP4, MSP5, and MSP6 was compared between low- and high-miR responders. Data were assessed for significance by nonparametric paired Wilcoxon test (A-C) or nonparametric Mann-Whitney test ( $\mathbf{D}$ and $\mathbf{E}$ ). Box-and-whisker plots: boxes show 25th to 75th percentiles, whiskers extend to all data, line within box shows median; lowmiR responders $n=9$, high-miR responders $n=10 .{ }^{*} P<0.05$; ${ }^{* *} P<0.01$. ns, not significant.

Table 4) and included 2 genes that were differentially expressed prior to infection (IL2RA and RUNX1). Thus, low- and high-miR responders have distinct patterns of differential gene expression prior to infection.

Of the 17 mRNAs significantly upregulated in the low-responder group prior to infection, 11 are predicted targets for at least one of the miRs in our 3-miR signature (miR15a-3p, miR30c-5p, and miR30e-5p) (microRNA.org, Good mirSVR score, Conserved miR). A comparison of the expression levels of the 3-signature miRs and the $17 \mathrm{mRNAs}$ significantly upregulated prior to infection revealed a positive correlation in all cases, reaching statistical significance for $73 \%$ (37 of 51$)$ of the correlations $(P<0.05$, Spearman correlation). By day 7 of infection $79 \%$ of correlations were negative, with only $10 \%$ reaching statistical significance (Table 5 list of correlation analysis; Figure 6 sample graphical correlation for 3 mRNAs predicted as targets for miR15a-3p, miR-30c, and miR-30e).

Taken together, these data suggest that low- and high-miR responders can be distinguished prior to infection by the expression of a signature pattern of miR and mRNA in their peripheral blood. 
A
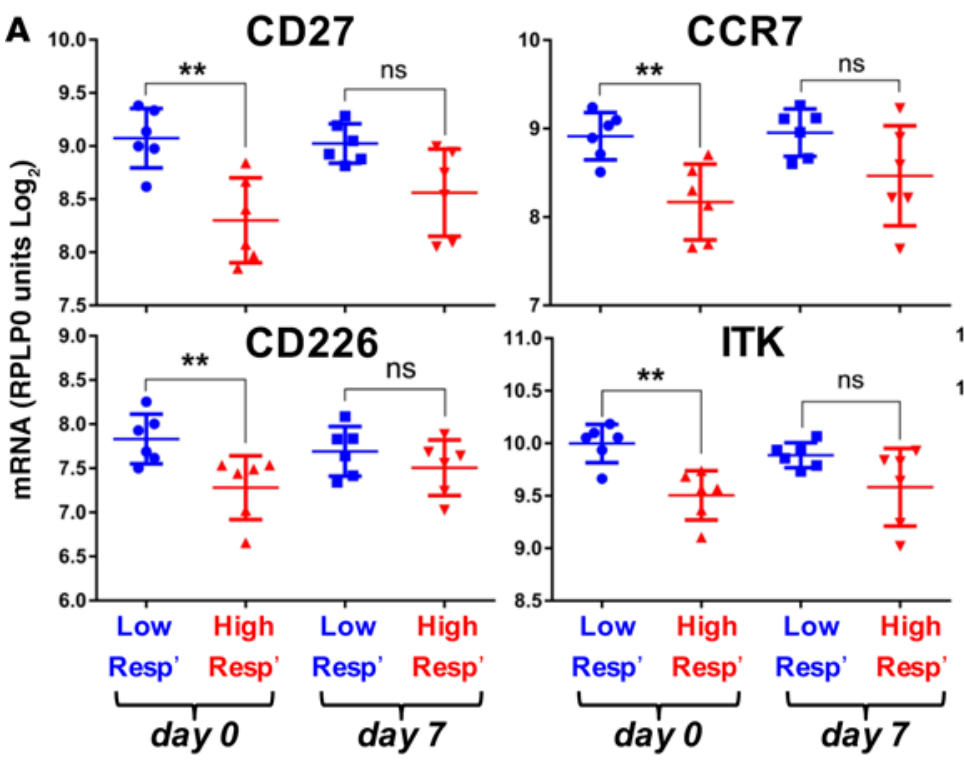

CCR7
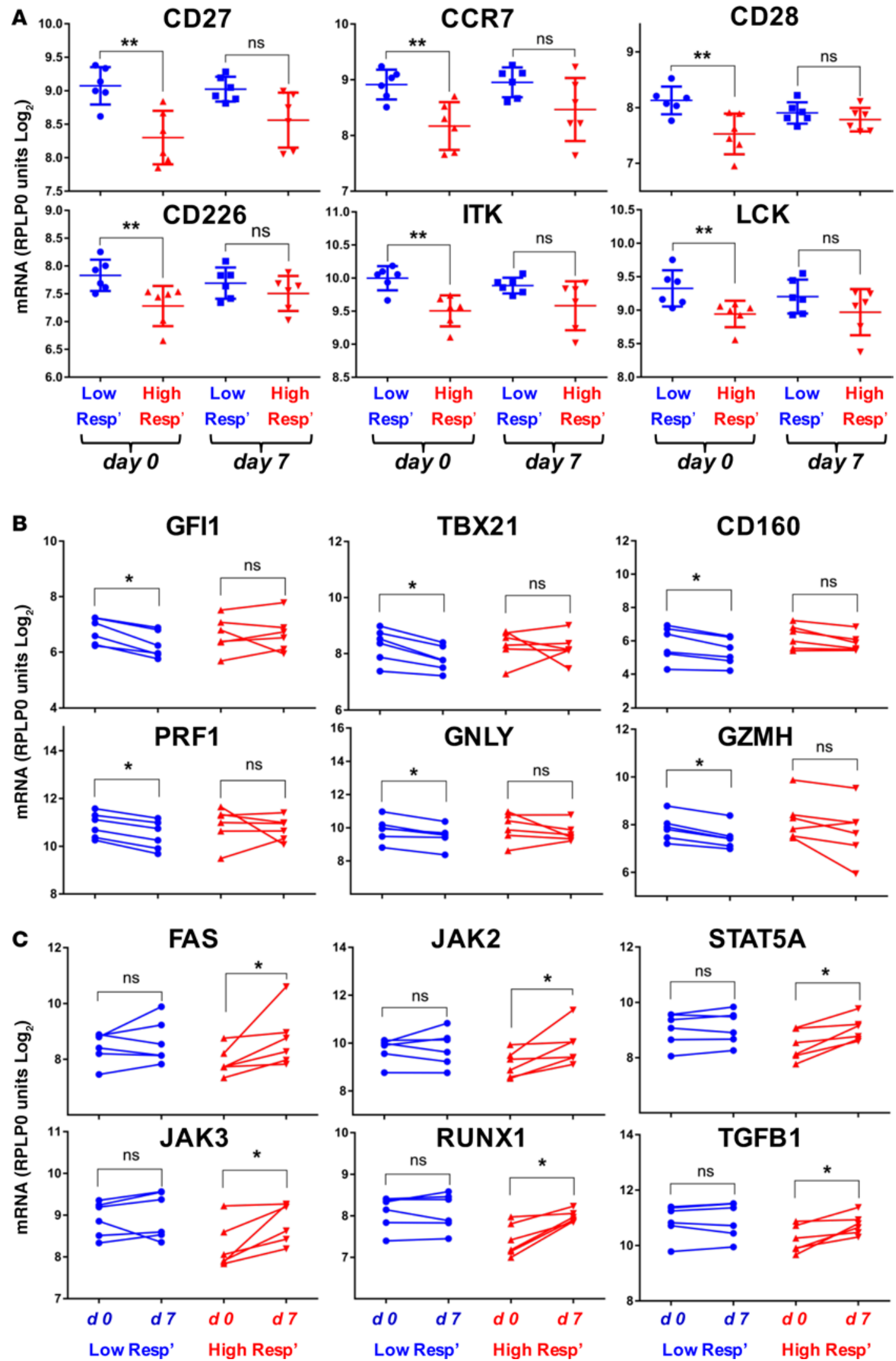

Figure 5. mRNA expression defines low- and high-miR responder groups prior to and during infection. mRNA expression in whole blood was determined for a set of 135 genes using NanoString in 12 volunteers taken prior to (d0), and on day 7 (d7) of infection the from high-and low-miR responder groups. (A) The top 6 differentially expressed genes between the low- and high-miR responders groups by $P$ value (Mann-Whitney test, mean and SD are indicated). Paired analysis of changes in mRNA expression for individuals between $\mathrm{d} 0$ and $\mathrm{d} 7$ of infection (Wilcoxon test), (B) the top 6 regulated genes for the low-miR responders and (C) for the high-miR responders (by $P$ value). ${ }^{*} P<0.05$; ${ }^{* *} P<0.01$. ns, not significant. 


\section{Discussion}

Herein, we utilized a controlled human malaria infection to assess immune responses in otherwise healthy malaria-naive volunteers experimentally infected with $P$. falciparum. We used targeted miR profiling to measure the expression of $84 \mathrm{miRs}$ known to be associated with $\mathrm{T}$ cell and B cell activation and identified a subset of 71 reproducibly detected prior to and during infection. When we compared miR expression levels in individuals prior to and during infection, we found a distinct dichotomy across all volunteers: approximately $50 \%$ upregulated the expression of these miRs (high-miR responders), whereas the remaining volunteers displayed no change or downregulated the set of miRs (low-miR responders). From this set of 71 miRs, we were able to define a 3-miR signature (miR15a-3p, miR30c-5p, and miR30e-5p) that distinguished high-miR responders from low-miR responders.

Using linear regression analysis, we identified a significant inverse relationship between the miR responses and parasite growth within the volunteers over the first 7 days of infection. High-miR responders had lower parasite burden, while low-miR responders had higher parasite burden; thus, the high-miR responders were better able to control the growth of the $P$. falciparum parasite during primary infection. Of note, our parasite burden measurement was based on the area under the parasite concentration curve from the first 7 days of infection, assuming that all cohorts received the same starting inoculum concentration. We found that the distribution of the volunteers into high- and low-miR responders was cohort independent, excluding the idea that this dichotomy was driven by a parasite inoculum effect. Nevertheless, the influence of the parasite infective dose on interindividual variability in immune response to infection remains to be addressed.

Concordant with the negative association with parasite burden, compared with low-miR responders, high-miR responders displayed increased numbers of recently activated and proliferating $\mathrm{CD}^{+} \mathrm{T}$ cells in their peripheral blood over the first 7 days of infection. Further supporting this dichotomy, high-miR responders (but not low-miR responders) displayed significant increases in IgG antibody titer against total blood-stage parasite extract in their plasma 28 days after infection. A more comprehensive investigation of the antibody response showed that high-miR responders had significantly increased IgG1, IgG3, and IgM anti-merozoite responses compared with low responders. These observations are important, as IgG1 and IgG3 are cytophilic antibody subclasses that function against merozoite antigens by fixing complement to the merozoite surface, inhibiting invasion (25) and enhancing opsonic phagocytosis (26). Moreover, cytophilic subclass antibodies (IgG1 and IgG3) against MSP1 and MSP2 have been associated with protection from malaria in longitudinal cohort studies in children $(23,24,27)$. These data demonstrate that high$\mathrm{miR}$ responders showed lower parasite growth rates during the first week of infection and were immunologically more reactive to infection, with enhanced effector $\mathrm{T}$ cell and antibody responses. Taken together, our observations suggest that high-miR responders are likely to become immune to symptomatic disease more rapidly than the low responders. The association between cellular and humoral immune responses was further established with the observation that only high-miR responders exhibited significant positive correlation between activated $\mathrm{CD} 69^{+} \mathrm{CD} 4^{+} \mathrm{T}$ cells and antigen-specific antibody responses. Interestingly, correlations are primarily for antigen MSP2 (and for 1 of 2 MSP1 proteins), but not for the other antigens MSP3, MSP4, MSP5, or MSP6. It is known that MSP1 and MSP2 are more prominent components of the initial response to malaria infection, and are generated after low levels of exposure, whereas other antigens require more exposure (28). An alternative explanation may be that MSP2 responses, particularly MSP2specific IgG responses, are due to germinal center responses, rather than extrafollicular response of the other IgM responses against the other MSP antigens (29).

We further investigated the miR dichotomy by evaluating the expression of 135 genes associated with effector immune responses, and identified a set of 17 genes whose expression was significantly increased prior to infection in the low-miR responder group when compared with the high responders. Over the first 7 days of infection, all of these significant differences were lost (except for 1 gene) and paired analysis of whole-blood mRNA from day 0 to day 7 of infection confirmed that low-miR responders downregulated mRNAs, while high responders tended to upregulate mRNAs. Although none of the statistical analyses resulted in significant Bonferroni-corrected $P$ values, a large proportion (11 out of 17 ) of the differentially expressed genes between low- and high-miR responders at day 0 are predicted targets for at least one of the miRs in our 3-miR signature, suggesting these miR/mRNA associations are more the result of a global shift in immune status between individuals rather than being driven by 1 or 2 particular genes. Additionally, the pattern of gene expression observed in low responders prior to infection, including increased 
Table 5. Correlation of miR and mRNA expression levels. Expression levels of the 3-miR signature (miR15a-3p, miR30c-5p, and miR30e-5p) and the set of mRNAs shown to be significantly regulated between the high- and low-miR responder groups prior to infection (above in

Table 2) were correlated at d0 and d7 (Spearman correlation, 6 volunteers from each of the high- and low-responder groups)

\begin{tabular}{|c|c|c|c|c|c|c|c|}
\hline & & \multicolumn{3}{|c|}{ day 0} & \multicolumn{3}{|c|}{ day 7} \\
\hline \multirow[t]{2}{*}{ CCR7 } & 1/slope & 1.27 & 1.82 & 1.31 & 12.77 & -2.56 & -2.28 \\
\hline & target & & & & & & \\
\hline \multirow[t]{2}{*}{ CD226 } & 1/slope & 1.15 & 1.64 & 1.07 & -1.70 & -2.48 & -2.18 \\
\hline & $P$ & ns & ns & ns & ns & ns & ns \\
\hline \multirow{2}{*}{ CD27 } & $P$ & B & A & A & ns & ns & ns \\
\hline & target & & & & & & \\
\hline \multirow[t]{3}{*}{ CD28 } & 1/slope & 1.08 & 1.43 & 1.02 & -3.63 & -1.21 & -0.71 \\
\hline & $P$ & A & ns & A & ns & A & ns \\
\hline & target & $\sqrt{ }$ & & & $\sqrt{ }$ & & \\
\hline \multirow{2}{*}{ CD40LG } & $P$ & A & A & B & ns & ns & ns \\
\hline & target & & & & & & \\
\hline \multirow[t]{3}{*}{ CD96 } & 1/slope & 0.99 & 1.08 & 0.90 & -3.62 & -2.07 & -1.11 \\
\hline & $P$ & A & A & A & ns & ns & ns \\
\hline & target & & $\sqrt{ }$ & $\sqrt{ }$ & & $\sqrt{ }$ & $\sqrt{ }$ \\
\hline \multirow[t]{3}{*}{ ICOS } & 1/slope & 2.17 & 2.41 & 1.17 & -0.94 & -0.91 & -0.82 \\
\hline & $P$ & A & ns & ns & ns & A & A \\
\hline & target & $\sqrt{ }$ & & & $\sqrt{ }$ & & \\
\hline \multirow[t]{3}{*}{ IL2RA } & 1/slope & 1.07 & 1.88 & 1.13 & 3.00 & 3.95 & 3.23 \\
\hline & $P$ & A & ns & A & ns & ns & ns \\
\hline & target & & $\sqrt{ }$ & $\sqrt{ }$ & & $\sqrt{ }$ & $\sqrt{ }$ \\
\hline \multirow{2}{*}{ ITGA4 } & $P$ & A & A & B & ns & ns & ns \\
\hline & target & $\sqrt{ }$ & & & $\sqrt{ }$ & & \\
\hline \multirow[t]{3}{*}{ ITK } & 1/slope & 0.92 & 1.11 & 0.83 & 2.77 & -2.32 & -1.27 \\
\hline & $P$ & A & ns & A & ns & ns & ns \\
\hline & target & $\sqrt{ }$ & $\sqrt{ }$ & $\sqrt{ }$ & $\sqrt{ }$ & $\sqrt{ }$ & $\sqrt{ }$ \\
\hline \multirow[t]{3}{*}{ LCK } & 1/slope & 0.75 & 0.79 & 0.63 & -2.03 & -1.61 & -1.06 \\
\hline & $P$ & c & B & B & ns & A & ns \\
\hline & target & & & & & & \\
\hline \multirow[t]{3}{*}{ RUNX1 } & 1/slope & 0.99 & 1.30 & 0.98 & -4.56 & 28.58 & 2.45 \\
\hline & $P$ & A & A & A & ns & ns & ns \\
\hline & target & $\sqrt{ }$ & $\sqrt{ }$ & $\sqrt{ }$ & $\sqrt{ }$ & $\sqrt{ }$ & $\sqrt{ }$ \\
\hline \multirow[t]{3}{*}{ S1PR1 } & 1/slope & 0.72 & 0.84 & 0.67 & 5.89 & -3.59 & -2.03 \\
\hline & $P$ & B & B & B & ns & ns & ns \\
\hline & target & & & $\sqrt{ }$ & & & $\sqrt{ }$ \\
\hline \multirow[t]{3}{*}{ SOCS5 } & 1/slope & 1.03 & 1.14 & 0.70 & -1.50 & -2.08 & -1.87 \\
\hline & $P$ & A & ns & A & ns & ns & ns \\
\hline & target & $\sqrt{ }$ & & & $\sqrt{ }$ & & \\
\hline
\end{tabular}

The slope and $P$ value of the correlation for each mRNA and miR, and whether the mRNA is a predicted target for the miR is shown $(\sqrt{ }) .{ }^{A} P<0.05 .{ }^{B} P<0.01$. c $P<0.001$. ns, not significant. 

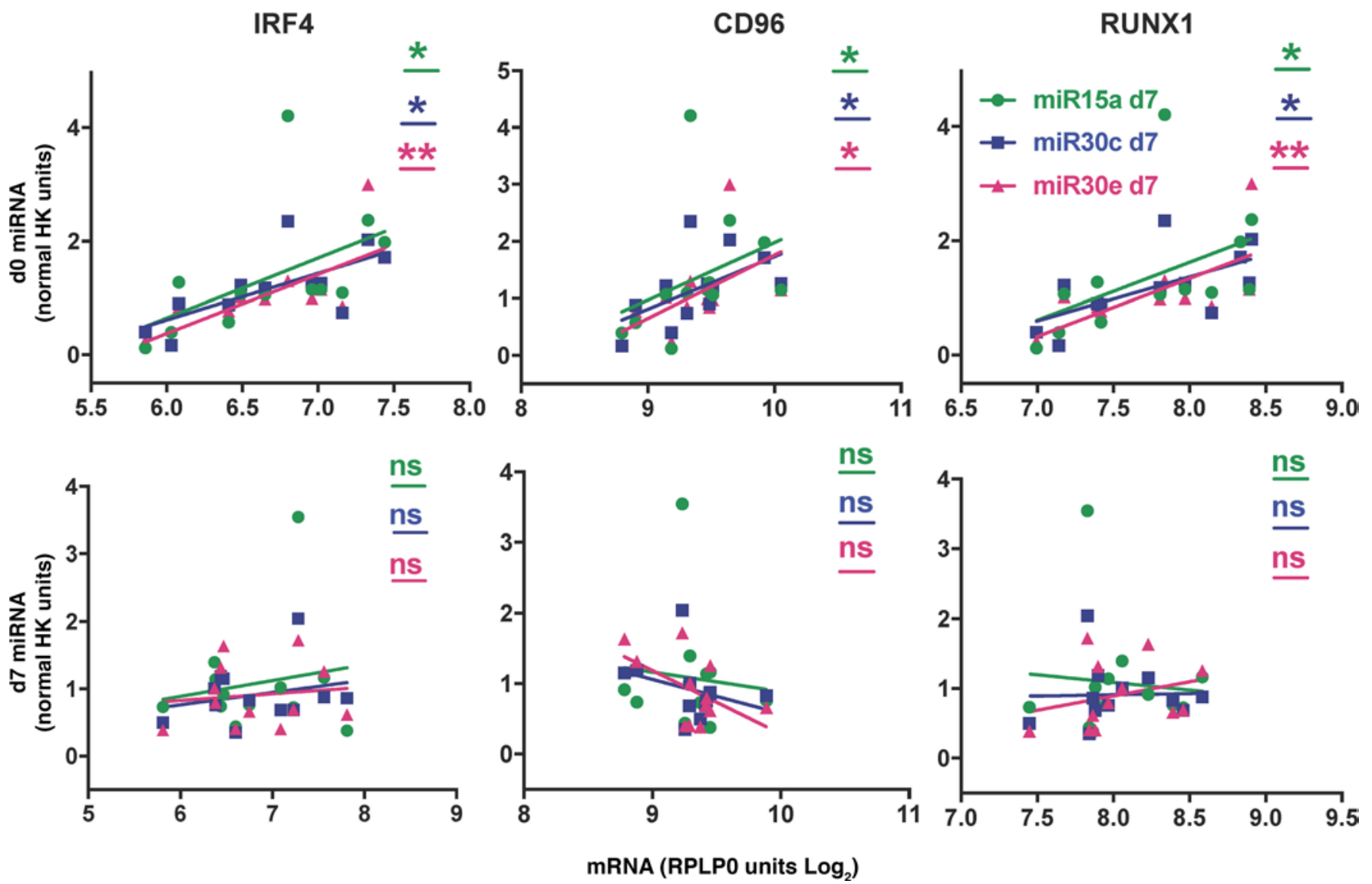

Figure 6. Strong positive correlations are evident for circulating miRs and mRNAs prior to infection. Expression levels of our 3-miR signature (miR15a-3p [green], miR30c-5p [blue], and miR30e-5p [pink]) and the set of mRNAs shown to be significantly regulated between the high-and low-miR responder groups prior to infection (above in Figure 5) were correlated at day 0 (d0) and d7 (Spearman correlation, 6 volunteers from each of the high- and low-responder groups). Graphical representation of correlations of 3 selected mRNAs predicted as targets for the 3-miR signature are shown. ${ }^{*} P<0.05 ;{ }^{* *} P<0.01$. ns, not significant.

CD27, CCR7, CD28, CD40LG, and IL7R, stands out as a hallmark signature of naive CD4 ${ }^{+} \mathrm{T}$ cells. Taken together, these results suggest that high responders may have been at a heightened immune state (with more activated memory $\mathrm{CD}^{+} \mathrm{T}$ cells over naive $\mathrm{CD} 4^{+} \mathrm{T}$ cells) prior to infection.

Our results are unexpected and our interpretation in the context of interindividual variability is that the Gaussian spread of responses to infection comprises 2 major groups (high- and low-miR responders), meaning that the major determinates are binary and not a continuum. To the best of our knowledge, this is the first such observation of a dichotomy in human immune response to infection. Moreover, most of the covariables we measured (parasite growth rate, cellular responses, and antibody response) would appear independently as normally distributed and would be measured in terms of increases from pre- to postinfection. However, the binary nature of the miR response (with either increasing or decreasing expression) allows us to view our dataset in a manner which would otherwise not be apparent.

Interindividual variability in the human response to infection with microbes is a phenomenon first recognized over a century ago. Although several lines of evidence provided the foundation for the idea, the work of Charles Nicolle and his report of inapparent infections provided the first solid evidence (5, 6). Today, the existence of interindividual variability and the notion that the underlying mechanisms comprise the sum of many factors such as individual differences in human genotype, past exposure, concurrent infections, and nutritional status; as well as variability in the genotype and dose of the infecting microbe are widely accepted (7). The net result of these factors is observed at the population level as a spectrum of disease states following infection with a given organism. The spectrum of disease often follows a Gaussian distribution where the majority may be mildly ill, while a small number will show no clinical symptoms and some will die. Understanding the mechanisms of interindividual variability in humans is of great interest because it could allow targeted strategies to help those at greater 
risk of serious illness or death. Nevertheless, it remains technically difficult to gain this understanding, partly due to the multifactorial underpinnings mentioned above, but also due to the technical and ethical constraints of performing scientific experiments in humans.

In the case of malaria, the clinical outcomes following Plasmodium infection are characteristically diverse and fit this pattern of interindividual variability. For naturally exposed individuals, dramatic variations in disease severity are observed, where symptoms range from very mild to rapid death. This diversity also applies to the rate at which individuals gain clinical immunity. Understanding how responses to Plasmodium infection develop is complex, and most observations of human immune responses to Plasmodium have been made in subjects from endemic areas, where previous exposure, infective dose, developing immunity, genetic variability of parasite and host, and parasitic coinfections are ever-present confounders. Models of controlled human malaria infection provide a unique opportunity to investigate the development of primary immune responses to Plasmodium infection in a highly controlled setting, where many of the potential confounders can be excluded.

The existence of a strong dichotomy in the development of immunity to blood-stage Plasmodium infection revealed by our controlled human malaria infection study has important practical implications. Indeed, our data suggest that approximately $50 \%$ of a malaria-naive population will mount an impaired immune response following infection with Plasmodium. This may explain the diversity and difficulties associated with naturally acquired immunity, as well as the demonstrated variable efficacy of malaria vaccines in clinical trials (30). Interestingly, the reported efficacy of the leading malaria vaccine, RTS,S, in phase 2 a studies in malaria-naive volunteers was typically $40 \%$ to $60 \%$ (31) and in phase 3 evaluation in children aged 5 to 17 months at first vaccination was $55.1 \%$ (32). We acknowledge that extrapolation of our findings and proving a dichotomous predisposition will be difficult in naturally exposed populations, as immune responses are cumulative and influenced by age, genetics, and coinfections. Furthermore, in CHMI studies, although many confounders can be accounted for, the clinical outcomes and development of immunity that would have eventuated had the infection been allowed to continue without drug treatment remain unknown; however, this could be partly addressed in future studies by a reinfection challenge of previously infected subjects. The use of controlled infection studies and studies of human responses to vaccination (33) coupled with recent advances in the field of systems biology (34) provides unique and valuable opportunities to dissect host-pathogen responses at an unprecedented level of detail to inform our understanding of human immunity and vaccine responsiveness.

In conclusion, we reveal the existence of a strong dichotomy in host immunity associated with the control of blood-stage malaria following primary exposure of malaria-naive human subjects. Individuals with increased expression of a defined 3-miR signature generated a higher frequency of activated and proliferating $\mathrm{T}$ cells and were better able to control parasite growth during infection, and developed a broader, more robust antibody response that was characterized by induction of cytophilic subclasses. These dichotomous responses were evident as early as day 4 of infection. Overall, our results suggest a predisposition in the ability of malaria-naive humans to develop effective immunity to malaria. These findings have important implications for vaccine development and for understanding the mechanisms driving interindividual variability in immune responses to malaria and other pathogens. Moreover, miRs and/or mRNA signatures that can effectively discriminate between high- and low-miR responders prior to infection would represent excellent biomarkers to predict vaccine nonresponsiveness and identify at-risk groups.

\section{Methods}

Sample collection and processing. Inoculum preparation, volunteer recruitment, infection, monitoring, and treatment were performed as described previously (11). In brief, healthy malaria-naive individuals underwent induced blood-stage malaria inoculation with 1,800 viable $P$. falciparum 3D7-parasitized erythrocytes, and parasite levels were measured at least daily by qPCR (see below) and treated with antimalarial drugs at day 7 or 8 of infection. Blood samples from 21 volunteers (from 4 independent cohorts) were collected prior to infection, 4 days, 7 days, and $28 \pm 3$ days after inoculation. Whole blood was collected in lithium heparin Vacutainers (BD Biosciences) for flow cytometric analysis and peripheral blood mononuclear cell (PBMC) isolation or in PAXgene blood RNA tubes (BD Biosciences) for RNA analysis. Plasma was collected from the lithium heparin whole-blood samples according to standard 
procedures, snap frozen in dry ice, and stored at $-70^{\circ} \mathrm{C}$. PBMCs were isolated from whole blood using Ficoll (Sigma-Aldrich) and cryopreserved in liquid nitrogen in 50\% DMSO, 40\% RPMI, and 10\% FCS according to standard procedures. A summary of subjects and cohorts used for the different assays is provided in Supplemental Table 3.

Determination of kinetics of parasitemia and parasite growth. Parasite levels over the time course of infection were determined using a consensus $P$. falciparum species-specific qPCR assay, as described previously (35). Parasite levels were measured once daily from day of infection and then twice daily once PCR-positive until treatment on day 7 or day 8 . All samples were batch tested in triplicate. The total parasite burden over the first 7 days of infection was defined as the AUC of the log-transformed parasite levels measured using the trapezoid rule. Limit of detection was 64 parasites/ml (35).

Total RNA and miR extraction. Whole blood was collected in PAXgene blood RNA tubes and left at room temperature for a minimum of 3 hours to ensure complete cell lysis before storage at $-20^{\circ} \mathrm{C}$. On the day of extraction, PAXgene blood RNA tubes were thawed at room temperature for a minimum of 2 hours. Total RNA and miRs were extracted using the PAXgene blood miR kit (Qiagen) according to the manufacturer's instructions. Total RNA samples were eluted in RNAse-free water and stored at $-70^{\circ} \mathrm{C}$. RNA quality was assessed and concentration quantified using the NanoDrop 2000 (Thermo Fisher Scientific); all A260/280 ratios were between 1.9 and 2.2.

miR expression using miScript PCR Qiagen Array. The level of expression of 84 miRs was measured by RT-qPCR using the T-Cell and B-Cell Activation miScript PCR Array (Qiagen) according to the manufacturer's instructions. Briefly, total miRs were reverse transcribed using the miScript II RT Kit (Qiagen) from 500 ng of total RNA extracted from the PAXgene blood RNA tubes and diluted 1:4 in RNAse-free water. miR qPCR was performed with 384-well plate Human T-Cell and B-Cell Activation miScript miRNA PCR Arrays (Qiagen) from $100 \mu 1$ of diluted template cDNA using QuantiTect SYBR Green PCR mix (Qiagen) and an ABI7900HT real-time PCR cycler (Life Technologies) according to the manufacturers' instructions. Cycling conditions were $95^{\circ} \mathrm{C}$ for 15 minutes (hot-start Taq DNA polymerase activation), 40 cycles at $95^{\circ} \mathrm{C}$ for 15 seconds (denaturation), $55^{\circ} \mathrm{C}$ for 30 seconds (annealing), and $60^{\circ} \mathrm{C}$ for 30 seconds (extension). Relative quantification during infection compared to prior to infection was calculated with the ddCt value method (36), using the mean Ct value of 5 selected reference small endogenous RNAs included in the miScript PCR Array which showed minimum variation across samples (SNORD61, SNORD68, SNORD95, SNORD96A, and RNU6-2). Fold-change expression values were then normalized to whole-blood lymphocyte concentration based on complete blood counts.

miR expression using Taqman microRNA assays. The level of expression of miR15a-3p, miR30c-5p, and miR30e-5p and the 2 small nuclear RNAs RNU44 and RNU48 as endogenous controls was measured from total RNA samples extracted from the PAXgene blood RNA tubes using Taqman microRNA assays (Life Technologies). Briefly, 100-300 ng of RNA was reverse transcribed using the Taqman microRNA RT kit (Life Technologies) and a RT primer pool containing all Taqman microRNA assays at $0.2 \times$ final concentration according to the manufacturer's instructions. qPCR was then performed from $0.25 \mu 1$ of RT product using Taqman microRNA assays at $1 \times$ final concentration, the Taqman Universal Master Mix II No AmpErase UNG (Life Technologies), and an ABI 7900 HT real-time PCR cycler (Applied Biosystems) according to the manufacturers' instructions. Cycling conditions were $95^{\circ} \mathrm{C}$ for 10 minutes (DNA polymerase heat activation), 40 cycles at $95^{\circ} \mathrm{C}$ for 15 seconds, and $60^{\circ} \mathrm{C}$ for 1 minute (amplification). Relative miR quantification during infection compared to prior to infection was calculated with the ddCt value method (36), using the mean Ct value of RNU44 and RNU48 as a reference (37).

$m R N A$ expression using NanoString. Expression of 135 immune-related genes known to be involved in $\mathrm{T}$ cell and B cell responses was assessed using a custom 135-plex nCounter custom codeset of genes involved in immune recognition, survival, migration, adhesion, cytokine/chemokine secretion, activation, differentiation, and exhaustion, as reported previously (38). nCounter codeset hybridization was performed as per manufacturer's instructions using $100 \mathrm{ng}$ of total RNA from the same samples assayed for miR expression (above). Samples were processed using the NanoString GEN2 Prep Station and data acquired using the nCounter Digital Analyzer (NanoString Technologies). Data were normalized to the mean RPLP0 expression levels of 24 samples and processed using nSolver (NanoString Technologies) using the recommended settings. The highest negative control value for each sample was subtracted from each count of that sample and any values of zero or less were considered as zero. All genes reported herein were greater than zero. The data were Log2 normalized for statistical analysis. 
Ex vivo phenotyping of lymphocytes from whole blood by flow cytometry. Whole blood collected in lithium heparin Vacutainers was lysed and fixed with BD FACS lysing solution (BD Biosciences) and lymphocytes permeabilized with BD FACS permeabilizing solution 2 (BD Biosciences) according to the manufacturer's instructions. Cells were then stained with $50 \mu$ of FACS staining buffer (PBS supplemented with $0.5 \%$ FCS and 4 mM EDTA) containing anti-human CD4-AF488 (BD Biosciences, clone RPA-T4), anti-human CD8-APC-H7 (BD Biosciences, clone SK1), anti-human CD19-PE Cy7 (Biolegend, clone HIB19), anti-human CD69-AF700 (Biolegend, clone FN50), anti-human CD38-APC (Biolegend, clone HB7), anti-human Ki67-PerCpCy5.5 (Biolegend, clone Ki-67), and $1 \mu \mathrm{l}$ of human Fc receptor blocking solution (Human TruStain FcX, Biolegend) for 30 minutes at room temperature. Cells were washed and resuspended in FACS staining buffer before acquisition on an LSRFortessa 4 instrument (BD Biosciences) using Diva software. FlowJo software version 6.0 was used for gating.

Parasite-specific antibody responses. Parasite-specific antibody titer was measured in triplicate plasma samples using an ELISA assay against $P$. falciparum schizont extract. Maxisorp NUNC-immuno 96-well plates (Thermo Fisher Scientific) were precoated with $50 \mu 1$. falciparum schizont extract at $1 \times 10^{6}$ parasitized cells/ $\mathrm{ml}$ in carbonate buffer $\left(1.59 \mathrm{~g} / 1 \mathrm{Na}_{2} \mathrm{CO}_{3}, 2.93 \mathrm{~g} / 1 \mathrm{NaHCO}_{3}, \mathrm{pH} 9.6\right)$ overnight at $4^{\circ} \mathrm{C}$. Plates were washed with PBS, blocked with $5 \%$ skim milk powder in PBS $/ 0.05 \%$ Tween for 2 hours at $37^{\circ} \mathrm{C}$, and washed with PBS $/ 0.05 \%$ Tween. Plasma samples diluted $1: 100$ in $0.5 \%$ skim milk powder in PBS $/ 0.05 \%$ Tween were added at $50 \mu \mathrm{l}$ per well and plates incubated for 2 hours at $37^{\circ} \mathrm{C}$ before washing in PBS $/ 0.05 \%$ Tween. Secondary antibody (HRP-conjugated goat anti-human IgG, Chemicon) diluted 1:5,000 in 0.5\% skim milk powder in $\mathrm{PBS} / 0.05 \%$ Tween was added at $50 \mu \mathrm{l}$ per well, and plates were incubated for 45 minutes at room temperature followed by a wash in PBS $/ 0.05 \%$ Tween. For merozoite antigen-specific responses, the following antigens were coated at $0.5 \mu \mathrm{g} / \mathrm{ml}$ in $50 \mu \mathrm{l}$ of PBS overnight at $4^{\circ} \mathrm{C}$ : MSP1-42 (42-kDa fragment of MSP1), a gift from Carole Long at the NIH (His-tagged produced in E. coli) (39); MSP2 (3D7 allele, full length, produced in E. coli), a gift from Robin Anders, LaTrobe University, Melbourne, Victoria, Australia (40); MSP3 (expressed in E. coli as previously described (41); MSP4 and MSP5 (3D7, expressed in E. coli), a gift from Ross Coppel at Monash University, Melbourne, Victoria, Australia (42, 43); and MSP6 generated as previously described (44). Plates were washed with PBS $/ 0.05 \%$ Tween, blocked for 2 hours in 1\% casein/PBS, and then incubated with $50 \mu \mathrm{l}$ of plasma sample in duplicate diluted 1:100 in $0.1 \%$ casein/PBS for 2 hours. For total IgG, antibodies were detected with anti-human total IgG-HRP conjugated (Zymed) at 1:2,000 dilution in 0.1\% casein. For IgM and IgG subclass responses, secondary antibodies (IgM clone HP6083, IgG1 clone HP6069, IgG2 clone HP6002, IgG3 clone HP6050 [all from Thermo Fisher Scientific], and IgG4 clone HP6025 from Sigma-Aldrich) were diluted 1:2,000 in $0.1 \%$ casein/PBS, incubated for 1 hour, washed, and detected with goat anti-mouse-HRP (Chemicon) at 1:2,000 in 0.1\% casein/PBS. For all ELISAs, development was performed using the TMB substrate solution (Sigma-Aldrich) and the Stop reagent for TMB substrate solution (Sigma-Aldrich), or hydrochloric acid according to the manufacturer's instructions. Reaction was stopped after approximately 5 minutes and absorbance immediately measured at $450 \mathrm{~nm}$ using VersaMax tunable microplate reader (Molecular Devices). Antibody titers were measured as the average absorbance of sample wells, with the absorbance of blank wells (no plasma sample added) subtracted.

Statistics. Statistical analyses were performed using GraphPad Prism Software, version 6. Normality was assessed using D'Agostino and Pearson Omnibus normality test and showed that most datasets were not normally distributed. Therefore, paired datasets were compared using the nonparametric Wilcoxon test, while unpaired datasets were compared using the nonparametric Mann-Whitney test. $P$ values less than 0.05 were considered significant and 2-tailed analyses were performed. Correlation between miR expression and parasite burden was assessed using Pearson's correlation test. All other correlations were assessed using the Spearman's rank correlation coefficient test.

Study approval. Experimental infection of malaria-naive healthy volunteers was undertaken at QPharm Pty Ltd. (Brisbane, Australia); (clinical trial numbers: NCT02453581, NCT02453581, ACTRN12613000565741) with written informed consent under protocols approved of the QIMR Berghofer Medical Research Institute Human Research Ethics Committee (QIMRB-HREC).

\section{Author contributions}

JG Burel, SHA, JG Beeeson, and DLD designed the experiments. JG Burel, PLG, SHA, and MJB performed the experiments. CL generated antigens MSP3 and MSP6. JG Burel, SHA, and MJB analyzed the data. JSM undertook the clinical trial and provided the clinical samples. JG Burel, SHA, and DLD wrote the manuscript with contribution from all authors. 


\section{Acknowledgments}

Funding for the clinical trials was provided by Medicines for Malaria Venture (MMV) from grants awarded by the Wellcome Trust and Bill and Melinda Gates Foundation. The laboratory work supported by the National Health and Medical Research Council (NHMRC) Australia (Program Grant 1037304). We thank Joerg Moehrle and Tim Wells from MMV, Geneva for facilitating access to study samples from the clinical trials. JG Burel was supported by a UQ International scholarship from the University of Queensland. DLD was supported by an NHMRC Principal Research Fellowship. JSM was supported by an NHMRC Practitioner Fellowship and a Government of Queensland Clinical Research Fellowship. MJB was supported by an NHMRC Early-Career Fellowship. The Burnet Institute is supported by the NHMRC Australia Infrastructure for Research Institutes Support Scheme and the Victorian State Government Operational Infrastructure Support grant. We thank the Q-pharm staff who conducted the human infection studies, in particular Suzanne Elliot, Nannette Douglas and Gem Mackenroth; Paul Griffin; as well as Katharine Trenholme, Fiona Amante, and Silvana Sekuloski for their critical role in the study management. We gratefully acknowledge Thomas Watkins and John Miles for their role in designing the NanoString custom codeset. We also thank the Flow cytometry and Imaging Facility at QIMR Berghofer Medical Research Institute for assistance with flow cytometry. The authors declare no competing financial interests. The funders had no role in the study design, data collection and analysis, decision to publish, or preparation of the manuscript.

Address correspondence to: Denise L. Doolan, James Cook University, McGregor Road, Smithfield, QLD 4878, Australia. Phone: 61.7.4232.1492.Email: Denise.Doolan@jcu.edu.au.

JG Burel's present address is: La Jolla Institute for Allergy and Immunology, San Diego, California, USA.

1. WHO. World Malaria Report 2015. Geneva, Switzerland: WHO; 2015. http://www.who.int/malaria/publications/world-malaria-report-2015/report/en/. Accessed July 13, 2017.

2. Comstock GW, Livesay VT, Woolpert SF. The prognosis of a positive tuberculin reaction in childhood and adolescence. $A m J$ Epidemiol. 1974;99(2):131-138.

3. Dye C, Floyd K. Tuberculosis. In: Jamison DT, et al., eds. Disease Control Priorities in Developing Countries. Washington, DC, USA: World Bank Publications; 2006: Chapter 16.

4. Taubenberger JK, Morens DM. 1918 Influenza: the mother of all pandemics. Emerging Infect Dis. 2006;12(1):15-22.

5. Garnham PCC. Charles Nicolle and inapparent infections. Am J Trop Med Hyg. 1977;26:1101-1104.

6. Nicolle, C. 2013. Destin des maladies infectieuses. Paris, France: Presses Électroniques de France, ISBN 9791022300292; 2013.

7. Casanova JL. Human genetic basis of interindividual variability in the course of infection. Proc Natl Acad Sci U S A. 2015;112(51):E7118-E7127.

8. Collins WE, Jeffery GM, Roberts JM. A retrospective examination of reinfection of humans with Plasmodium vivax. Am J Trop Med Hyg. 2004;70(6):642-644.

9. Collins WE, Jeffery GM. A retrospective examination of secondary sporozoite- and trophozoite-induced infections with Plasmodium falciparum: development of parasitologic and clinical immunity following secondary infection. Am J Trop Med Hyg. 1999;61(1 supp1):20-35.

10. Walther $\mathrm{M}$, et al. Innate immune responses to human malaria: heterogeneous cytokine responses to blood-stage Plasmodium falciparum correlate with parasitological and clinical outcomes. J Immunol. 2006;177(8):5736-5745.

11. McCarthy JS, et al. A pilot randomised trial of induced blood-stage Plasmodium falciparum infections in healthy volunteers for testing efficacy of new antimalarial drugs. PLoS One. 2011;6(8):e21914.

12. Engwerda CR, Minigo G, Amante FH, McCarthy JS. Experimentally induced blood stage malaria infection as a tool for clinical research. Trends Parasitol. 2012;28(11):515-521.

13. Chen CZ, Schaffert S, Fragoso R, Loh C. Regulation of immune responses and tolerance: the microRNA perspective. Immunol Rev. 2013;253(1):112-128.

14. Lindsay MA. microRNAs and the immune response. Trends Immunol. 2008;29(7):343-351.

15. Tambyah PA, et al. microRNAs in circulation are altered in response to influenza A virus infection in humans. PLoS One. 2013;8(10):e76811.

16. Reynoso R, et al. MicroRNAs differentially present in the plasma of HIV elite controllers reduce HIV infection in vitro. Sci Rep. 2014;4:5915.

17. Fu Y, Yi Z, Wu X, Li J, Xu F. Circulating microRNAs in patients with active pulmonary tuberculosis. J Clin Microbiol. 2011;49(12):4246-4251.

18. Miotto P, et al. miRNA signatures in sera of patients with active pulmonary tuberculosis. PLoS One. 2013;8(11):e80149.

19. Burel JG, Apte SH, Groves PL, Klein K, McCarthy JS, Doolan DL. Reduced plasmodium parasite burden associates with $\mathrm{CD} 8^{+} \mathrm{CD}^{+} \mathrm{T}$ cells displaying cytolytic potential and impaired IFN- $\gamma$ production. PLoS Pathog. 2016;12(9):e1005839.

20. Beeson JG, Drew DR, Boyle MJ, Feng G, Fowkes FJ, Richards JS. Merozoite surface proteins in red blood cell invasion, immunity and vaccines against malaria. FEMS Microbiol Rev. 2016;40(3):343-372.

21. Gilson PR, et al. Identification and stoichiometry of glycosylphosphatidylinositol-anchored membrane proteins of the human 
malaria parasite Plasmodium falciparum. Mol Cell Proteomics. 2006;5(7):1286-1299.

22. Teo A, Feng G, Brown GV, Beeson JG, Rogerson SJ. Functional antibodies and protection against blood-stage malaria. Trends Parasitol. 2016;32(11):887-898.

23. Stanisic DI, et al. Acquisition of antibodies against Plasmodium falciparum merozoites and malaria immunity in young children and the influence of age, force of infection, and magnitude of response. Infect Immun. 2015;83(2):646-660.

24. Taylor RR, Allen SJ, Greenwood BM, Riley EM. IgG3 antibodies to Plasmodium falciparum merozoite surface protein 2 (MSP2): increasing prevalence with age and association with clinical immunity to malaria. Am J Trop Med Hyg. 1998;58(4):406-413.

25. Boyle MJ, et al. Human antibodies fix complement to inhibit Plasmodium falciparum invasion of erythrocytes and are associated with protection against malaria. Immunity. 2015;42(3):580-590.

26. Osier FH, et al. Opsonic phagocytosis of Plasmodium falciparum merozoites: mechanism in human immunity and a correlate of protection against malaria. BMC Med. 2014;12:108.

27. Stanisic DI, et al. Immunoglobulin G subclass-specific responses against Plasmodium falciparum merozoite antigens are associated with control of parasitemia and protection from symptomatic illness. Infect Immun. 2009;77(3):1165-1174

28. McCallum FJ, et al. Differing rates of antibody acquisition to merozoite antigens in malaria: implications for immunity and surveillance. J Leukoc Biol. 2017;101(4):913-925.

29. Kurosaki T, Kometani K, Ise W. Memory B cells. Nat Rev Immunol. 2015;15(3):149-159.

30. Clinical Trials Partnership. Efficacy and safety of RTS,S/AS01 malaria vaccine with or without a booster dose in infants and children in Africa: final results of a phase 3, individually randomised, controlled trial. Lancet. 2015;386(9988):31-45.

31. Cohen J, Nussenzweig V, Nussenzweig R, Vekemans J, Leach A. From the circumsporozoite protein to the RTS, S/AS candidate vaccine. Hum Vaccin. 2010;6(1):90-96.

32. Clinical Trials Partnership. First results of phase 3 trial of RTS,S/AS01 malaria vaccine in African children. N Engl J Med. 2011;365(20):1863-1875.

33. Querec TD, et al. Systems biology approach predicts immunogenicity of the yellow fever vaccine in humans. Nat Immunol. 2009;10(1):116-125.

34. Burel JG, Apte SH, Doolan DL. Systems approaches towards molecular profiling of human immunity. Trends Immunol. 2016;37(1):53-67.

35. Rockett RJ, et al. A real-time, quantitative PCR method using hydrolysis probes for the monitoring of Plasmodium falciparum load in experimentally infected human volunteers. Malar J. 2011;10:48.

36. Livak KJ, Schmittgen TD. Analysis of relative gene expression data using real-time quantitative PCR and the $2(-\Delta \Delta \mathrm{C}(\mathrm{T}))$ method. Methods. 2001;25(4):402-408.

37. Viprey VF, Corrias MV, Burchill SA. Identification of reference microRNAs and suitability of archived hemopoietic samples for robust microRNA expression profiling. Anal Biochem. 2012;421(2):566-572.

38. Proietti C, et al. Mining, visualizing and comparing multidimensional biomolecular data using the Genomics Data Miner (GMine) Web-Server. Sci Rep. 2016;6:38178.

39. Singh S, et al. Immunity to recombinant plasmodium falciparum merozoite surface protein 1 (MSP1): protection in Aotus nancymai monkeys strongly correlates with anti-MSP1 antibody titer and in vitro parasite-inhibitory activity. Infect Immun. 2006;74(8):4573-4580.

40. McCarthy JS, et al. A phase 1 trial of MSP2-C1, a blood-stage malaria vaccine containing 2 isoforms of MSP2 formulated with Montanide ISA 720. PLoS One. 2011;6(9):e24413.

41. Polley SD, et al. Plasmodium falciparum merozoite surface protein 3 is a target of allele-specific immunity and alleles are maintained by natural selection. J Infect Dis. 2007;195(2):279-287.

42. Wang L, Black CG, Marshall VM, Coppel RL. Structural and antigenic properties of merozoite surface protein 4 of Plasmodium falciparum. Infect Immun. 1999;67(5):2193-2200.

43. Marshall VM, Coppel RL. Characterisation of the gene encoding adenylosuccinate lyase of Plasmodium falciparum. Mol Biochem Parasitol. 1997;88(1-2):237-241.

44. Woehlbier U, Epp C, Hackett F, Blackman MJ, Bujard H. Antibodies against multiple merozoite surface antigens of the human malaria parasite Plasmodium falciparum inhibit parasite maturation and red blood cell invasion. Malar J. 2010;9:77. 